\title{
Case study on polymyositis
}

\section{Introduction}

The inflammatory myopathies are a group of acquired skeletal muscle diseases that includes polymyositis (PM), dermatomyositis (DM), and inclusion body myositis (IBM). PM is an idiopathic inflammatory myopathy clinically characterized by proximal muscle weakness, elevations of serum muscle enzymes and additionally in DM by skin abnormalities. PM have prevalence rates estimated at approximately 1 per 100,000 in the general population. There is a female to male predominance of about 2:1. The peak incidence in adults is between the ages of 40 and 50 .

Although the inciting event of PM is unknown, it has been postulated that some microvascular injury may lead to the release of muscle auto antigens, which are then presented to T-lymphocytes by macrophages in the muscle. T-lymphocytes that have been activated then proliferate and release cytokines such as interferon gamma and interleukin. Interferon gamma promotes further macrophage activation and release of mediators of inflammation such as IL-1 and tumour necrosis factor-alpha. The presence of auto aggressive inflammatory cells that surround, enter, and destroy morphologically normal appearing myofibres is the characteristic feature of PM. These inflammatory cells are composed largely of $\mathrm{CD} 8^{+}$Tcells and macrophages.

The main symptoms are:

1. Difficulty swallowing (dysphasia)

2. Difficulty speaking

3. Arthralgia

4. Fatigue

5. Shortness of breath

6. Pain in shoulder, upper arm, legs, neck.

The predominant symptom of PM is muscle weakness. Weakness is symmetric, affecting the proximal muscles of the extremities as well as the neck flexors. Main diagnostic test include:

Muscle Enzymes: Laboratory markers of muscle injury include elevations in the blood levels of CK, aldolase, AST, ALT, and LDH. CK elevations occur at some point in most PM patients, and CK is probably the most reliable enzyme to measure

Autoantibodies: A variety of auto antibodies may be present in the serum of patients with PM. Some of these, such as anti-nuclear antibodies (ANA) and antibodies to Ribo Nucleo Protein (RNP).

Magnetic resonance imaging (MRI): Can demonstrate early muscle inflammation in PM.

Muscle biopsy: Can demonstrate muscle fibres in various stages of inflammation, necrosis and regeneration, endomysial infiltration by mononuclear cells, capillary obliteration, endothelial cell damage, and increased amounts of connective tissue.

\section{Management of PM: general approach}

The goals of therapy are:
Volume I Issue I - 2017

\section{Sriram S, Christy Mary Varghese \\ Department of clinical pharmacy, University of Sri Ramakrishna Hospital Campus, India}

Correspondence: Sriram S, Department of clinical pharmacy, University of Sri Ramakrishna Hospital Campus, Coimbatore, India, Email visitram@yahoo.com

Received: April 22, 2017| Published: May 03, 2017

i) To improve muscle weakness

ii) To avoid the development of extra-muscular diseases of the vital organs.

Corticosteroids: Corticosteroids like prednisone are the first line of therapy for PM. The usual starting dose is $1 \mathrm{mg} / \mathrm{kg} /$ day of prednisone or its equivalent. This does is usually maintained for the first 6-8 weeks. The response to therapy should be assessed every 2-4 weeks by monitoring the proximal muscle strength, muscle enzyme levels, and patient functionality. After the initial 6-8 weeks, a slow taper of steroids should begin. Many rheumatologists start a steroid-sparing immunosuppressive agent at the time steroids are initiated, while others prefer to reserve these agents for patients who have clearly failed corticosteroid mono therapy.

Steroid-sparing drugs: In a steroid responsive patient, the goal is to attain the lowest dose of steroids that will adequately manage the disease. AZA (Azathioprine) is usually administered orally at a dose of $1.5-3.0 \mathrm{mg} / \mathrm{kg} /$ day. MTX (Methotrexate) may be administered orally, subcutaneously, or intramuscularly. MTX is given once a week at doses ranging from $10-40 \mathrm{mg}$.

Mycophenolate mofetil: It is administered orally in doses up to $3 \mathrm{~g} /$ day.

Calcineurin inhibitors: Cyclosporine A and tacrolimus have both shown some efficacy in the treatment of refractory PM. Cyclosporine $\mathrm{A}$ is administered orally in doses up to $150 \mathrm{mg}$ twice a day.

Intravenous immunoglobulin: In patients who are corticosteroid resistant, especially where there is rapidly progressive or lifethreatening progression, intravenous immunoglobulin (IVIG) may be helpful. The recommended initial dose is $2 \mathrm{~g} / \mathrm{kg} /$ month.

Rituximab (RTX): It is a monoclonal antibody against CD $20^{+}$ B-cells, which causes depletion of these cells for 6 months or longer. The optimal dose of RTX is to use $375 \mathrm{mg} / \mathrm{m}^{2}$, infused intravenously once a week for 4 weeks.

Cyclophosphamide (CTX): It is an alkylating agent that is toxic to lymphopoietic cells. Both T-cells and antibody producing B-cells are affected. This drug may be most useful in patients who have PM with interstitial lung disease. The drug may be given intravenously at 0.8 $1.0 \mathrm{~g} / \mathrm{m}^{2} /$ month for several months.

\section{Case discussion}

A Female patient of 40years was admitted in neurology ward with 
complaints of breathing difficulty, pain on both legs and neck pain. Patient was found to be hypokalemic. Based on the elevated serum creatine kinase (CK) levels and potassium levels, the patient was diagnosed to have polymyositis. Patient was admitted for 5 days and treated with Inj. Ivepred (Methyl Prednisolone) 500mg twice daily, T. Among (Amlodipine) 5mg once daily, Syp. Potklor (Potassium chloride) $10 \mathrm{ml}$ thrice daily, Inj. Deriphylline $110 \mathrm{mgthrice}$ daily $\mathrm{T}$. Aldactone (Spironolactone) $25 \mathrm{mg}$ twice daily (Table 1).

Table I ECG: Sinus tachycardia, abnormal rhythm

\begin{tabular}{lllll}
\hline Vital signs & Day I & Day 2 & Day 3 & Day 4 \\
\hline Blood pressure $(\mathrm{mm} \mathrm{Hg})$ & $120 / 80$ & $110 / 70$ & $120 / 80$ & $120 / 80$ \\
Temperature $(\mathrm{F})$ & 98.6 & 98.6 & 98.6 & 98.6 \\
Pulse (beats $/ \mathrm{min})$ & 88 & 88 & 86 & 86
\end{tabular}

\begin{tabular}{lll}
\hline Clinical investigations & Day I & Day 3 \\
\hline Creatine Kinase(U/L) & 4119 & 3040 \\
Potassium (meq/l) & 1.2 & 1.8 \\
\hline
\end{tabular}

\section{Conclusion}

Polymyositis is a life threatening condition if left untreated, can result in worsening of breathing and swallowing problems. PM is more common in females and most affected muscles are muscles of hips and thighs, upper arms, shoulder, neck. PM can also affect cardiac muscles causing inflammatory myopathy and muscles involved in breathing. The predominant clinical manifestation is proximal muscle weakness. There may be extra muscular involvement such as inflammatory arthritis, Raynaud's phenomenon, myocarditis, and interstitial lung disease. Serum muscle enzymes (CK) are usually elevated during periods of active disease. A variety of auto antibodies are often found in the serum of PM patients. Characteristic abnormalities are often seen on EMG and muscle MRI. Definitive diagnosis is established by muscle biopsy. The first line agent in treating PM is usually a steroid. Steroid especially Prednisolone is very effective in bringing inflammation under control, restoring the swallowing, breathing and hear functions. Life style modifications like intake of thickened fluids, eat softer ormashed foods, gentle physio exercises are to be followed to improve movement and decrease pain. ${ }^{1-6}$

\section{Acknowledgements}

None.

\section{Conflict of interest}

The author declares no conflict of interest.

\section{References}

1. Hill CL, Zhang Y, Sigurgeirsson B, et al. Frequency of specific cancer types in dermatomyositis and polymyositis: a population-based study. Lancet. 2001;357(9250):96-100.

2. Strauss KW, Gonzalez-Buritica H, Khamashta MA, et al. Polymyositisdermatomyositis: a clinical review. Postgrad Med J. 1989;65(765):437443 .

3. Zhang L, Wang GC, Ma L, et al. Cardiac involvement in adult polymyositis or dermatomyositis: a systematic review. Clin Cardiol. 2012;35(11):686-691.

4. Fathi M, Dastmalchi M, Rasmussen E, et al. Interstitial lung disease, a common manifestation of newly diagnosed polymyositis and dermatomyositis. Ann Rheum Dis. 2004;63(3):297-301.

5. Hengstman GJ, van Engelen BG. Polymyositis, invasion of nonnecrotic muscle fibres, and the art of repetition. British Medical Journal. 2004;329(7480):1464-1467.

6. Hajj-ali, Rula A. Polymyositis and Dermatomyositis. Merck Manual Home Edition; 2013. 